\title{
Transcriptomic and proteomic profiles of II YOU 838 (Oryza sativa) provide insights into heat stress tolerance in hybrid rice
}

\author{
Yan Wang ${ }^{1,2}$, Yang Yu ${ }^{1}$, Min Huang ${ }^{2}$, Peng Gao ${ }^{2}$, Hao Chen ${ }^{2}$, Mianxue Liu ${ }^{2}$, Qian Chen ${ }^{2}$, Zhirong Yang ${ }^{1}$, Qun \\ Sun ${ }^{\text {Corresp. } 1}$ \\ ${ }^{1}$ Key Laboratory of Bio-resource and Bio-environment of the Ministry of Education, College of Life Science, Sichuan University, Chengdu, Sichuan, China \\ 2 Key Laboratory of Irradiation Preservation of Sichuan Province, Sichuan Institute of Atomic Energy, Chengdu, Sichuan, China \\ Corresponding Author: Qun Sun \\ Email address: qunsun@scu.edu.cn
}

Heat stress is an increasing threat to rice production worldwide. To investigate the mechanisms of heat tolerance in hybrid rice and their contributions to rice heterosis, we compared the transcriptome of the hybrid rice II YOU 838 (II8) with the transcriptomes of its parents Fu Hui 838 (F8) and II-32A (II3) after heat stress at $42^{\circ} \mathrm{C}$ for $0,24,72$, and 120 $\mathrm{h}$. We also performed a proteomic analysis in 118 after heat stress at $42^{\circ} \mathrm{C}$ for $24 \mathrm{~h}$. The transcriptome data revealed time-dependent gene expression patterns under the heat stress conditions, and the heat stress response of 118 was greatly different from those of its parents. Gene ontology analysis of the differentially expressed genes that were clustered using k-means clustering showed that most of the up-regulated genes were involved in responses to stimuli, cell communication, and metabolic and transcription factor activities, whereas the down-regulated genes were enriched in photosynthesis and signal transduction. Moreover, 35 unique differentially abundant proteins, including a basic helixloop-helix transcription factor (bHLH96), calmodulin-binding transcription activator, heat shock protein (Hsp70), and chaperonin 60 (CPN60), were detected in the proteomic analysis of II8 under heat stress. The co-regulatory analysis revealed novel genes and pathways involved in heat tolerance, namely, ferredoxin-NADP reductase, peroxidases, mitogen-activated protein kinase kinase kinase, and heat shock factor (HSF)-Hsp network. Members of the Hsp and HSF families had over-dominant expression patterns in the hybrid compared with its parents, to help maintain the higher photosynthesis and antioxidant defense systems in the hybrid. Our study suggests that the complex HSF-Hsp regulatory network contribute to the heat tolerance of the hybrid rice. 


\section{Transcriptomic and proteomic profiles of II YOU 838 (Oryza sativa)}

2

3

4

5

6

7

8

9

10

11

12

13

14

15

16

17

18

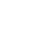

\section{provide insights into heat stress tolerance in hybrid rice}

Yan Wang ${ }^{1,2}$, Yang Yu ${ }^{1}$, Min Huang ${ }^{2}$, Peng $\mathrm{Gao}^{2}$, Hao Chen ${ }^{2}$, Mianxue $\mathrm{Liu}^{2}$, Qian $\mathrm{Chen}^{2}$, Zhirong Yang ${ }^{1} \&$ Qun Sun ${ }^{1}$

${ }^{1}$ Key Laboratory of Bio-resource and Bio-environment of the Ministry of Education, College of

Life Sciences, Sichuan University, Chengdu, Sichuan 610064, China

${ }^{2}$ Key Laboratory of Irradiation Preservation of Sichuan Province, Sichuan Institute of Atomic

Energy, Chengdu, Sichuan 610066, China

Corresponding author:

Qun Sun, Key Laboratory of Bio-resource and Bio-environment of the Ministry of Education, College of Life Sciences, Sichuan University, No. 29 Wangjiang Road, Chengdu 610064, China

ORCID: $\underline{\text { https://orcid.org/0000-0002-4372-8865 }}$

Tel: +86-28-8541-8810

Fax: $+86-28-8541-8810$

E-mail: qunsun@scu.edu.cn 
19 ABSTRACT

20 Heat stress is an increasing threat to rice production worldwide. To investigate the mechanisms

of heat tolerance in hybrid rice and their contributions to rice heterosis, we compared the transcriptome of the hybrid rice II YOU 838 (II8) with the transcriptomes of its parents Fu Hui 838 (F8) and II-32A (II3) after heat stress at $42^{\circ} \mathrm{C}$ for $0,24,72$, and $120 \mathrm{~h}$. We also performed a proteomic analysis in II 8 after heat stress at $42^{\circ} \mathrm{C}$ for $24 \mathrm{~h}$. The transcriptome data revealed timedependent gene expression patterns under the heat stress conditions, and the heat stress response of II8 was greatly different from those of its parents. Gene ontology analysis of the differentially expressed genes that were clustered using k-means clustering showed that most of the upregulated genes were involved in responses to stimuli, cell communication, and metabolic and transcription factor activities, whereas the down-regulated genes were enriched in photosynthesis and signal transduction. Moreover, 35 unique differentially abundant proteins, including a basic helix-loop-helix transcription factor (bHLH96), calmodulin-binding transcription activator, heat shock protein (Hsp70), and chaperonin 60 (CPN60), were detected in the proteomic analysis of II8 under heat stress. The co-regulatory analysis revealed novel genes and pathways involved in heat tolerance, namely, ferredoxin-NADP reductase, peroxidases, mitogen-activated protein kinase kinase kinase, and heat shock factor (HSF)-Hsp network. Members of the Hsp and HSF families had over-dominant expression patterns in the hybrid compared with its parents, to help maintain the higher photosynthesis and antioxidant defense systems in the hybrid. Our study suggests that the complex HSF-Hsp regulatory network contribute to the heat tolerance of the hybrid rice. 
40 Keywords: Hybrid rice; Flag leaf; Heat stress tolerance; Transcriptome; Proteome; Co-

41 regulatory network

\section{Introduction}

44 Global warming has led to an increase in extreme weather events in recent decades (Wang et al. 2019). Extreme weather conditions, such as high temperature and drought, have greatly affected rice production and food security worldwide. Cultivated rice (Oryza sativa) is the staple food for more than half of the world's population (Zhang et al. 2012b). O. sativa subsp. indica accounts for more than $70 \%$ of rice production globally (Zhang et al. 2016b). It is, however, very sensitive to high-temperature stress during almost all growth stages, particularly the flowering period (Wang et al. 2019; Zafar et al. 2018). Rice grain yields decline by $10 \%$ for each $1{ }^{\circ} \mathrm{C}$ increase in the minimum night time temperature during the growing period (Peng et al. 2004). Heat stress can cause irreversible damage by retarding plant growth, metabolic activities, spikelet fertility, and seed setting, thus reducing the rice production (Wang et al. 2019). Heat stress also may significantly suppress the photosynthetic rate, hormone levels, membrane stability, respiration, and primary and secondary metabolites (Zafar et al. 2018). At the cell level, heat shock induces thermo-tolerance responses and activates the expression of genes encoding heat shock proteins osmoprotectants to offset heat stress-induced biochemical and physiological changes (Hasanuzzaman et al. 2013). Consequently, understanding the molecular basis of heat tolerance will greatly contribute to the development of new strategies for improving heat tolerance in rice 
61 (Cheabu et al. 2018; Li et al. 2018b).

The tolerance of rice to heat stress is linked with the ability to perceive the high temperature stimulus and generate and transmit the signal, as well as with the expression of specific genes and production of metabolites, and the initiation of the antioxidative defense system (Anjum et al. 2016; Cao et al. 2008; Zafar et al. 2018). In response to sudden heat shock, a number of mechanisms and several compounds are produced to protect rice plants from stress. Heat stress damages the internal cellular environment, activates the heat tolerance machinery, and triggers the expression of heat shock genes. This initiates the production of Hsps, antioxidant enzymes, free radical scavengers, and osmoprotectants to detoxify reactive oxygen species (ROS) and recover the cell membrane so that cellular homeostasis is reestablished (Hasanuzzaman et al. 2013; Li et al. 2018a; Zhao et al. 2018). In transgenic plants overexpressing an Hsp gene, ROS scavenging and antioxidant enzyme activity increased under heat stress (Liu et al. 2018). The heat tolerance of rice is associated with complex regulatory networks, and the genes involved in the related pathways may respond differently at different time points in different tissues (Hasanuzzaman et al. 2013). RNA sequencing (RNA-Seq) is a powerful high-throughput technique that has been used to systematically investigate the molecular reactions by which rice responds and adapts to different heat stress environments (Sarkar et al. 2014; Zhang et al. 2012b; Zhang et al. 2013). Proteomic analyses of rice under heat stress conditions have been reported (Damaris et al. 2016; Gammulla et al. 2010; Zou et al. 2011). However, there are currently no published studies on the dynamic changes at the proteome and transcriptome levels during a prolonged heat stress regime in rice flag leaves, especially during the flowering stage. Although 
82 a heat shock response is a short-term reaction, heat stress usually lasts for a long time over all

83 rice developmental stages in the field. Previous studies focused mainly on changes at the

84

85

86

87

88 beginning of stress (Gammulla et al. 2010; Sarkar et al. 2014; Zhang et al. 2012b; Zou et al. 2011). In this study, a relatively longer heat shock response time was studied to obtain a more comprehensive understanding of the regulatory network involved in heat stress tolerance in rice.

Rice hybrids usually show strong heterosis, which is a complex multigenic trait that can be extrapolated as the sum total of physiological and phenotypic traits, including resistance to biotic and abiotic environmental stresses (Zhang et al. 2016b). To overcome the challenges presented by global warming, it is important to understand how rice hybrids perceive and respond to high temperatures. Hybrid rice II YOU 838 (II8), which was obtained from a cross between Fu Hui 838 (F8) and II-32A (II3), exhibits superior multiple agronomic traits including yield and adaptability, especially resistance to high temperatures. Thus, II8 has been cultivated widely in China (Su et al. 2013; Wang et al. 2016b). Although its resistance to heat stress has been confirmed in the field, the mechanism remains to be deciphered. Although many studies have been conducted on heat stress mechanisms using heat resistant materials and mutants, they are different from rice varieties such as II8 that are used in production (Han et al. 2018; Poli et al. 2013; Wang et al. 2016a; Zhang et al. 2018).

We compared the transcriptomic data of flag leaves from the hybrid II8 variety with the transcriptomic data of flag leaves from its parents during long-term heat stress by RNA-Seq. We also surveyed the mechanisms underlying the higher heat tolerance of II8 by transcriptomic and proteomic analyses. F8 is a mutation-containing variety derived from $O$. sativa subsp. indica 
103

104

105

106

107

108

109

110

111

112

113

114

115

116

117

118

119

120

121

122

123

MH63 (Deng et al. 2009). The indica varieties have high levels of genetic diversity; therefore,

we used the MH63 genome and proteome data as the references in this study (Song et al. 2018;

Zhang et al. 2016a; Zhang et al. 2016b). The combination of transcriptomic and proteomic

profiles provided insights into the mechanisms of heat tolerance in hybrid rice and their

contributions to heterosis.

\section{Materials \& Methods}

\section{Plant materials and heat treatment}

The hybrid rice line II8, along with the paternal F8 and maternal II3 lines, were cultivated in the experimental field in Sichuan Province, China. The field management followed essentially normal agricultural practice. At the heading stage (108 days after sowing), the plants were transferred to a growth chamber maintained at $22^{\circ} \mathrm{C} / 32^{\circ} \mathrm{C}(13-\mathrm{h} / 11-\mathrm{h}$ dark/light cycle) with $80 \%$ humidity and light intensity of $600 \mu \mathrm{mol} \mathrm{m} \mathrm{m}^{-2} \mathrm{~s}^{-1}$ for $48 \mathrm{~h}$ to allow the plants to adapt to the growth chamber. Then, the control flag-leaf samples $(0 \mathrm{~h})$ were collected from the II8, F8, and II3 lines at 19:00 (i.e. after 11 -h light at $32^{\circ} \mathrm{C}$ and before 13 -h dark at $32^{\circ} \mathrm{C}$ ) and frozen immediately in liquid nitrogen. On the first flowering day, the II8, F8, and II3 lines were heat treated at $32^{\circ} \mathrm{C} / 42^{\circ} \mathrm{C}(13-\mathrm{h} / 11-\mathrm{h}$ dark/light cycle $)$ for $120 \mathrm{~h}$ during the blossoming days. The flag leaves of the three lines were harvested at $24 \mathrm{~h}, 72 \mathrm{~h}$, and $120 \mathrm{~h}$, and frozen immediately in liquid nitrogen. Light, humidity, water, and fertilizer conditions in the growth chamber were all the same during the 168-h experimental period. Each sample had three biological replicates during the flowering stage. 


\section{Total RNA and total protein isolation}

126 Total RNA was extracted from the flag leaves of the three lines using TRIzol (Invitrogen,

127 Carlsbad, CA, USA) and treated with DNase I (Thermo Fisher Scientific, Waltham, MA, USA)

128 following the manufacturer's instructions. The purity of the RNA was assessed by determining

129 the absorbance levels at $260 \mathrm{~nm}$ and $280 \mathrm{~nm}$ using a spectrophotometer. RNA integrity was

130 checked using an Agilent 2100 Bioanalyzer (Agilent, Santa Clara, CA, USA). The RNA samples

131 from three independent biological replications were mixed equally and used for RNA-Seq. The

132 same RNA samples from three biological replicates were used independently for quantitative

133 real-time RT-PCR (qPCR).

Total proteins was extracted from the flag leaves of II8 plants that were subjected to the

same heat stress conditions at $0 \mathrm{~h}$ and $24 \mathrm{~h}$ using a two-step precipitation/extraction method

(Jiang et al. 2012). Three independent biological replicates were used.

\section{Library sequencing and data processing}

The sequencing libraries of II8 and the parental lines were constructed using digital gene

expression profiling techniques as described in a previous study (Tao et al. 2012). The RNA-Seq

was carried out on an Illumina HiSeq 2000 platform (Beijing Genomics Institution, Shenzhen,

China). The transcript levels were quantified using the transcripts per million (TPM) methods

(Zhang et al. 2012a). The same RNA samples from II8 flag leaves also were analyzed using a

deeper RNA-Seq method. Briefly, the RNA-Seq libraries were constructed as described in a 
145 previous study (Xing et al. 2017). The expression level of each gene was normalized to

146 fragments per kilobase per million (Trapnell et al. 2010). The Minghui 63 (MH63) reference

147 genome sequence (GenBank accession: LNNK00000000) was downloaded from the Rice

148 Information Gateway (http://rice.hzau.edu.cn) (Song et al. 2018; Zhang et al. 2016b). A fold

149 change (Dias et al.) with an absolute value of $\log 2 \mathrm{FC}>1$ and a stringent false discovery rate

$150<0.001$ were set as the thresholds to define differentially expressed genes (DEGs). A gene

151 ontology (GO) enrichment analysis was performed using the singular enrichment analysis in

152 agriGO (Tian et al. 2017) with indica as the reference background

153 (http://bioinfo.cau.edu.cn/agriGO/index.php). Hypergeometric tests were performed using the

154 default parameters and Benjamini-Hochberg corrected $p$-values. Hierarchical clustering, k-means

155 clustering, and the STRING database (v10.5) (Szklarczyk et al. 2017) analysis were performed to

156 determine co-regulatory patterns. The network was visualized in Cytoscape (Baryshnikova 2016),

157 with genes as nodes and interactions as links (edges) between the nodes. The RNA-Seq data have

158 been deposited in the NCBI Sequence Read Archive (https://www.ncbi.nlm.nih.gov/sra) under

159 accession number SRP168528.

160

161

qPCR analysis

162 The qPCR analysis was carried out using the SYBR Green 2.0× PCR reaction mix (TaKaRa,

163 Japan). The primer pairs were designed using NCBI Primer-Blast (Ye et al. 2012) and

164 synthesized by Invitrogen (Table S1). The relative expression levels of all the candidate genes

165 were normalized against the expression levels of three internal controls, rice ACTIN1 gene

PeerJ reviewing PDF | (2019:08:39987:1:0:NEW 27 Oct 2019) 
166

167

168

169

170

171

172

173

174

175

176

177

178

179

180

181

182

183

184

185

186

MH03g0618400 (Jain et al. 2006), MH01g0139700, and MH02g0177100, as described

previously (Vandesompele et al. 2002). For each gene, three technical replicates and three independent biological replicates were used at each sampling time point.

\section{Two-dimensional polyacrylamide gel electrophoresis (2D-PAGE) and data analysis}

The 2D-PAGE was performed as described by Jiang et al (Jiang et al. 2012). After

electrophoresis, the gels containing the three biological replicates were silver stained (Sinha et al. 2001) and scanned at an optical resolution of 300 dpi using a UMAX scanner (UTA-1100, GE

Healthcare, USA). The digitalized images were analyzed using ImageMaster 2D Platinum software (v5.0, GE Healthcare, USA). The spot volume was normalized as a percentage of the total volume of all the spots in a gel (vol\%). Spot abundance (vol\%) ratios $>2$ with $p<0.05$ were set as the thresholds to identify differentially abundant proteins (DAPs). Protein spots of interest were excised from the gels, digested, and then subjected to nanoelectrospray ionization followed

by tandem mass spectrometry (MS/MS) in an LTQ Orbitrap Velos (Thermo Fisher Scientific, San Jose, CA, USA) coupled online to high-performance liquid chromatography instrument.

Proteins were identified by searching the MH63 reference proteome, which was downloaded from the Rice Information Gateway using the Mascot peptide search engine v2.3.02 (Matrix Science, London, UK).

\section{Results}

Three indica rice varieties, II8 and the parental lines F8 and II3, were heat treated for $120 \mathrm{~h}$. 
187 Visible damage to the flag leaves was detected after $120 \mathrm{~h}$ of heat stress (Fig. 1A). Under the 188 same heat stress conditions, the damage to II8 flag leaves was the least serious, followed by F8

189 flag leaves; the damage to II3 flag leaves was the most obvious. This result indicated that II8 was 190 more tolerant to the high temperature than its parents. In a previous study, physiological and biochemical adaptations, such as chlorophyll content, and peroxidase, superoxide dismutase, and catalase activity, were higher in II8 flag leaf than in the flag leaves of the paternal lines under heat-stress conditions (Wang et al. 2016b). The differences that we observed in the flag leaf phenotypes confirmed the appropriateness of using II8 and the parental lines for the transcriptome analysis.

Differential gene expression patterns in II8 and the parental lines in response to different

durations of heat stress

The DEGs were detected using the thresholds FC $\geq 2$-fold and false discovery rate $<0.001$. In the three comparisons ( 0 h vs 24 h, 0 h vs 72 h, 0h vs 120 h), more DEGs was up-regulated than down-regulated DEGs in all three lines (Fig. 1B). This suggests that under the heat stress conditions, more genes were switched on than switched off to promote thermo-tolerance. A total of 4016, 3073, and 3596 DEGs were detected in II8, F8, and II3, respectively. The higher number of DEGs that were found in the hybrid may be related to the higher heat resistance of II8 compared with its parents. We consider that changes in the transcriptome are mainly the results of long-term adaptive events associated with heat stress tolerance. 
208

209

210

211

212

213

214

215

216

217

218

219

220

221

222

223

224

225

226

227

228

980 down-regulated) detected in the hybrid after $24 \mathrm{~h}$ of heat stress were greater than the corresponding numbers in its parents. However, after $72 \mathrm{~h}$ of heat stress, the total number of DEGs (868) and the exclusive number of DEGs (180 up-regulated and 203 down-regulated) in the hybrid were smaller than the corresponding numbers in its parents (Fig. 1B). The 24-h and 72-h time points may be key transitional times in the response of II8 to the high temperature conditions. Similarly, after $120 \mathrm{~h}$ of heat stress, the exclusive number of DEGs (426 up-regulated and 235 down-regulated) in the hybrid was smaller than the corresponding numbers in its parents.

We detected 1653 DEGs that were exclusively up-regulated at one or more of the time points and 1542 of them were over-dominant genes. Among them, 213, 1315, 327, and 640 were up-regulated at $0 \mathrm{~h}, 24 \mathrm{~h}, 72 \mathrm{~h}$, and $120 \mathrm{~h}$, respectively (Table S2). The expression levels of the over-dominant genes were higher in the hybrid than in its parents (Wei et al. 2009). The overdominant effects of genes can combine to promote heterosis (Shapira \& David 2016). Using a minimal TPM value of 10 and an absolute value of $\log 2 \mathrm{FC} \geq 10$, we detected 73 markedly differentially expressed over-dominant genes that were associated with heat stress responses in the hybrid (Table S3). Approximately $26 \%$ of them (19 genes) were annotated as expressed proteins or hypothetical proteins with unknown functions. This suggests that genes associated with heat tolerance in the hybrid are a rich area for further investigations. The expression levels of the small heat shock protein (sHsp) genes MH06g0146900 and MH02g0661300, and the Hsp90 activator gene MH06g0689400 were greatly increased under long-term heat stress. Nine transcription factor (TF) genes encoding TFs in the WRKY, zinc finger, MYB, bHLH, and other 
229

230

231

232

233

234

235

236

237

238

239

240

241

242

families, as well as genes encoding mitogen-activated protein kinase kinase kinase (MAP3K;

MH01g0546600) and peroxidase 1 (MH05g0063600) were greatly up-regulated. In addition, genes encoding transposons, retrotransposons, transporters, protein kinases, and protein phosphatases were strongly induced by long-term heat stress. These highly up-regulated genes are involved in a very large proportion of the life processes, indicating that heat tolerance is associated with complicated regulatory networks in the hybrid.

Although short-term heat stress responses have been studied in rice, our results indicate that a large number of genes are positively involved in the heat responses during long-term heat stress.

\section{Differential gene expression patterns in II8 in response to different durations of heat stress}

Details of the heat tolerance mechanisms in the hybrid are not known; therefore, the gene expression levels in II8 flag leaves subjected to long-term heat stress during the flowering stage were studied using a deeper RNA-Seq method. The deeper transcriptome profiles of the hybrid were consistent with the digital gene expression profiling results. The number of up-regulated genes was higher than the number of down-regulated genes (Fig. 2A). Most of the DEGs underwent the highest number of transcriptional changes after $24 \mathrm{~h}$ of heat stress. Many of the heat-responsive genes exhibited transient differential expression but reverted to the unstressed levels after $72 \mathrm{~h}$ of heat stress. A Venn diagram analysis showed that 586 significantly regulated genes were continuously differentially expressed after $24 \mathrm{~h}, 72 \mathrm{~h}$, and $120 \mathrm{~h}$ of heat stress (Fig. 2B). A hierarchical clustering analysis of these 586 DEGs was performed to identify the co- 
250

251

252

253

254

255

256

257

258

259

260

261

262

263

264

265

266

267

268

269

270

regulatory pattern after long-term heat stress in the hybrid (Fig. 2C; Table S4). The analysis

showed that more genes were continuously up-regulated than continuously down-regulated. The

most significantly up-regulated genes were those encoding 3-ketoacyl-CoA synthase 11

(MH06g0569700), heat stress transcription factor B-2c (OsHsfB2c; MH09g0436500),

anthocyanidin 5,3-O-glucosyltransferase (MH03g0749700), transposon and retrotransposon

proteins (MH06g0215900, MH02g0167600, MH01g0656500, MH07g0247300,

MH04g0213900), Hsps (MH03g0171000, MH08g0491200, MH02g0624500), glutathione $S$ -

transferase (MH01g0791100), MYB (MH12g0137600), histone deacetylase 19

(MH02g0116000), and L-type lectin-domain containing receptor kinase (MH07g0035000), as

well as unknown hypothetical proteins (MH09g0263100, MH03g0211100, MH04g0309000).

\section{Expression pattern changes in II8 with different durations of heat-stress}

Because the changes in genome-wide gene expression under heat-stress conditions were complicated, the k-means clustering analysis method was used to investigate the expression pattern. The 5284 DEGs, which were identified in II8 flag leaves at least at one time point, formed 10 clusters (Fig. 3). Cluster A contained DEGs that were continuously down-regulated;

Clusters B and C contained DEGs that were continuously up-regulated; Clusters D-G contained DEGs that showed early up-regulation and then partial decrease; and Clusters $\mathrm{H}-\mathrm{J}$ contained DEGs that showed early down-regulation before reversion. The k-means clustering results show that the DEGs involved in the heat stress response followed a temporal regulatory pattern.

To determine the processes that were significantly affected by heat stress, a GO enrichment 
271

272

273

274

275

276

277

278

279

280

281

282

283

284

285

286

287

288

289

290

291

analysis of the DEGs in the early up- and down-regulated clusters, as well as the continuous upand down-regulated clusters was performed using agriGO (Tian et al. 2017). The results showed that most of the up-regulated genes were involved in cell communication (GO:0007154), response to stress (GO:0006950), response to abiotic stimulus (GO:0009628), response to external stimulus (GO:0009605), response to biotic stimulus (GO:0009607), response to endogenous stimulus (GO:0009719), response to stimulus (GO:0050896), metabolic process (GO:0008152) and transcription factor activity (GO:0003700), whereas the continuously downregulated genes were involved in photosynthesis (GO:0015979) and signal transduction (GO:0007165) (Fig. 4; Table S5). The GO terms in the response to stimulus pathway included response to stress, abiotic stimulus, external stimulus, endogenous stimulus, heat, oxidative stress, and biotic stimulus. The genes that were assigned these significant GO terms were modulated by long-term heat stress and are likely to be related to the heat tolerance of the hybrid.

\section{Identification of TFs in II8 that responded to long-term heat stress}

Among the 5284 DEGs in the hybrid, 160 encoded TFs belonging to $41 \mathrm{TF}$ families, including the WRKY (21 genes), MYB (16 genes), NAC (15 genes), AP2-EREBP (9 genes), bHLH (7 genes), bZIP (7 genes), and heat shock factor (HSF; 5 genes) families. Additionally, 77 of these

TF genes were more highly differentially expressed (absolute value of $\log 2 \mathrm{FC}>2$ ), either up- or down-regulated, at least at one of the time points (Table S6). The heat-responsive TFs encoded by these genes might work in isolation or act synergistically to regulate the expression levels of a large number of genes. 
(21 genes). WRKY TFs provide enhanced heat and drought tolerance, and function upstream of

294

295

296

297

298

299

300

301

302

303

304

305

306

307

308

309

310

311

312

the HSF-Hsp regulon (Li et al. 2011; Sarkar et al. 2014). All the WRKY-encoding genes were

up-regulated, except MH05g0036100. The transcript levels of MH01g0673800, MH05g0036100,

MH05g0538600, and MH08g0380600 were higher in the hybrid than in the parental lines after

$120 \mathrm{~h}$ of heat stress (Table S7). Members of the HSF family, HsfA4d (MH05g0501600), HsfA2b

(MH07g0091300), and HsfB1 (MH09g0368500) were remarkably induced in the hybrid during

the long-term heat treatment. The transcript levels of MH05g0501600 and MH09g0368500 were

up-regulated early in the hybrid and were higher than their levels in both parents after $24 \mathrm{~h}$ of

heat stress. Members of the bZIP family, MH06g0591100 and MH07g0509000, were up-

regulated in the hybrid on exposure to heat stress and were much higher than in the parental lines

after $24 \mathrm{~h}$ of heat treatment. The bZIPs have been found to trigger a heat response by unfolded

protein, leading to the expression of genes involved in endoplasmic reticulum quality control

under heat stress conditions (Kim et al. 2018; Lu et al. 2012; Takahashi et al. 2012).

Members of the MYB family, MH07g0545300, MH08g0419700, and MH09g0309700 also

more highly expressed in the hybrid than in the parental lines under long-term heat stress. The

transcript level of MH09g0309700 was higher in the hybrid than in the parental lines during the

120-h heat treatment. The bHLH family members, MH08g0482900 and MH03g0648500, were

up-regulated during the long-term heat stress and were highest in the hybrid after $24 \mathrm{~h}$ of heat

stress. The NAC family members, MH07g0041200 and MH12g0038200, the FAR1 family

member MH01g0445100, the CPP family member MH06g0213800, and the AP2-EREBP family

Peer) reviewing PDF | (2019:08:39987:1:0:NEW 27 Oct 2019) 
313 member MH10g0429500 were all remarkably induced in the hybrid during the long-term heat

314 stress. The transcript levels of all these genes were higher in the hybrid than in the parental lines,

315 especially after $24 \mathrm{~h}$ of heat stress.

316

317 Validation of gene expression

318 To validate the RNA-Seq results, eight genes (MH03g0686900, MH03g0260900,

MH03g0018700, MH04g0704000, MH07g0536400, MH10g0025400, MH03g0762100,

320

MH05g0501600) were selected randomly for independent validation by qPCR. All eight genes

321

were successfully amplified. As shown in Fig. 5A-H, the expression trends of the genes

322

determined by qPCR were in good agreement with the trends of the TPM data from the II8 and

323

parental lines. The transcripts levels of the selected genes in II 8 differed greatly from those of its

parents. A comparison between expression levels of the DEGs obtained by qPCR and the

fragments per kilobase per million data obtained by RNA-Seq in II8 flag leaves after different

durations of heat stress is shown in Fig. S1. The expression of all the selected genes obtained by

qPCR and by RNA-Seq were in good accord.

\section{Identification of DAPs under heat-stress conditions}

Hybrid flag leaves were heat treated for $0 \mathrm{~h}$ and $24 \mathrm{~h}$ and used to investigate the heat stress

responses at the proteome level. A total of 58 protein spots with $\mathrm{FC} \geq 2$-fold $(p<0.05)$ in all three

replicate samples were considered to be DAPs (Fig. S2). Among them, 35 were unique gene

products; 7 had increased abundance and 28 had decreased abundance after 24 h hear stress. The 
33435 unique DAPs were functionally annotated with GO terms (Table 1; Table S8). The most

335 enriched GO terms were response to stimulus, response to oxidative stress, response to abiotic

336 stimulus, and photosynthesis. The DAPs with significantly decreased abundance in response to

337 heat stress were disease resistance protein RGA1 (MH11g0587400), SNF1-related protein kinase

338 regulatory subunit gamma-like PV42a (MH02g0063200), bHLH96 (MH09g0379400), and

339 ferredoxin-dependent glutamate synthase (MH07g0519600). The DAPs with significantly

340 increased abundance in response to heat stress were Hsp70 (MH02g0644700), CPN60

341 (MH10g0328900), pentatricopeptide repeat-containing protein (MH05g0279300), and

342 calmodulin-binding transcription activator 2 (MH03g0091100). Two disease resistance proteins

343 (MH11g0587400 and MH12g0410900), which are sensors for biotic and abiotic stresses, also

344 were detected among the DAPs.

\section{Combined analysis of transcriptome and proteome data}

347 The transcriptome and proteome results shared a high rate of coincidence, with 18 of the 35

348 identified DAPs having the same abundance patterns (increased or decreased) as the

349 corresponding DEG expression levels (up- or down-regulated) (Table 1). The protein-protein

interaction networks of these DEGs/DAPs were predicted using the STRING database and the

353 shown in Table S7. The network contained one large and three small subclusters. The genes in 
355 kinase activity, and response to stimulus. The genes in the other three subcultures were most

356 strongly enriched in GO terms related to binding; metabolic process; and regulation and

357 photosynthesis.

358 There were nine hub genes in the network that had more than 10 edges; namely, HSP71.1

359 (MH03g0171000), Hsp81-1 (MH08g0491200), HsfB1 (MH09g0368500), CPN60

360 (MH10g0328900), HsfA4d (MH05g0501600), photosystem I reaction center subunit

361 (MH09g0391200), ferredoxin-dependent glutamate synthase (MH07g0519600), alcohol

dehydrogenase (MH11g0112300), and flavonoid 3'-monooxygenase (MH10g0172900). More

than $100(25 \%)$ of the 419 edges were involved in the HSF-Hsp network, including HsfA4d,

HsfB1, HsfB2c (MH09g0436500), Hsp81-1, Hsp70s, and sHsps in the cluster D (Fig. 6).

Members of the HSF family, HsfA4d, HsfB1, and HsfB2c were highly connected with

serine/threonine-protein kinase (MH01g0492100) and L-type lectin-domain containing receptor

kinase (MH04g0024800, MH07g0035000). Two Hsp70 family genes (MH03g0018700,

MH03g0171000) were highly connected with CPN60 and TFs. There were three sHsps

(MH02g0661300, MH02g0624500, MH06g0146900) in the co-regulatory network, which

indicated that sHsps play key roles in responses to heat stress. Li and Liu (Li \& Liu 2019)

reported that sHsps were highly conserved and might have similar functions in mediating the

responses of barley plants to heat stress. Members of the Hsp and HSF families were important

nodes with abundant connection points in the co-regulatory network. This indicated that the

HSF-Hsp network play important roles in the heat tolerance of II8.

While several genes in our co-regulatory network matched genes previously identified in 
376 rice seedlings (Sarkar et al. 2014), the overall topologies of the rice networks are dissimilar

377 because different sets of input genes were in the co-regulatory analyses. The hierarchical

378 clustering analysis of the 117 node genes revealed that the majority had over-dominant

379 expression patterns in the hybrid, especially after $24 \mathrm{~h}$ of heat stress (Fig. S3). These genes likely represent a heat-tolerance strategy in the hybrid during exposure to long-term heat stress.

\section{Discussion}

Rice hybrids have multiple resistance mechanisms to biotic and abiotic stresses, especially

resistance to high temperatures, and each of these mechanisms contributes, to some extent, to

heterosis (Feng et al. 2015; Hochholdinger \& Baldauf 2018). In this study, we performed

genome-wide transcriptome and proteome analyses of the hybrid rice II8, which is widely

cultivated in China, to gain a fundamental understanding of heat tolerance mechanisms of hybrid

rice and their contributions to heterosis. Moreover, heat stress induced early response genes that

exhibited transient expression but reverted later to unstressed levels. Therefore, the long-term

heat response period increased our understanding of the molecular basis of heat tolerance in rice.

Our results were not consistent with the observations of Sarkar et al. (2014) who found that

many genes were down-regulated after $1 \mathrm{~h}$ of heat stress in 1 month old rice plants. The

differences in the results may be because different samples were used. Compared with the

parental lines, more II8 genes showed changed expression levels in response to heat stress. This

suggested that the hybrid may have a more efficient heat stress response than its parents. The 
397

398

399

400

401

402

403

404

405

406

407

408

409

410

411

412

413

414

415

416

417

complementation between the parental genomes. These differences in the transcriptomes may

explain the superior heat tolerance of the hybrid.

To understand the relationships among the DEGs/DAPs in more detail and to detect

possible critical pathways, a co-regulatory analysis was conducted on the genes/proteins

identified in the hybrid. In the heat tolerance co-regulatory network, more genes were switched

on than switched off after long-term exposure to heat stress. Those increased at the protein and

up-regulated at the transcript levels included Hsp70s (MH03g0018700, MH02g0644700) and

sHsps (MH03g0686900, MH02g0661300, MH02g0624500). The interaction between Hsp70 and

the unfolded protein (its substrate) is regulated by co-chaperones, including sHsps (Awad et al.

2008). Under the same heat stress conditions, the changes in Hsp70 and sHsps transcript levels in

II8 were greater than those in the parental lines (Fig. 5). Hsp70 regulates the function HsfA1

through direct interactions, and represses HsfA1 activity (Hahn \& Scharf 2011). In the hybrid,

Hsp70 was up-regulated and OsHsfA1 (MH03g0762100) was down-regulated over the long-term

heat stress period. Unlike the parental lines, in the hybrid, OsHsfA4d (MH05g0501600) and

HsfB1 (MH09g0368500) were remarkably induced, especially at 24 h. OsHsfA and OsHsfB sub-

families were shown to have important functions in activating the cellular protection system and

heat-tolerance in rice (Zhang et al. 2012b). HSFs, which activate heat-stress responses, are

released from association with, and inhibition by, the Hsp70 and Hsp90 chaperones because the

chaperones bind to the misfolded proteins that result from heat stress (Zhu 2016). The Hsps,

including sHsp, Hsp90, and Hsp70, and their co-chaperones, function in preventing protein

denaturation and maintaining protein homeostasis. The HSF-Hsp regulatory network may be

PeerJ reviewing PDF | (2019:08:39987:1:0:NEW 27 Oct 2019) 
418 involved in controlling heat stress responses (Hahn \& Scharf 2011; Sarkar et al. 2014). We

419 hypothesize that the HSF-Hsp network is a main contributor to the heat tolerance of the hybrid.

420 A wild relative of cultivated $O$. sativa was shown to maintain high levels of the protective

421 proteins Hsp70, Hsp90, and CPN60, as well as a high photosynthetic rate to make it heat tolerant

422 (Scafaro et al. 2010). In the hybrid, the transcript and protein levels of CPN60 (MH10g0328900)

423 were increased under heat stress, and the rate of increase was greater than in the parental lines.

424 The folding of the ribulose-1,5-bisphosphate carboxylase/oxygenase (Rubisco) large subunit was

425 mediated by the cylindrical chloroplast CPN60 and its co-factor CPN20 (Wilson \& Hayer-Hartl

426 2018). Consistent with the observation by Gammulla et al (2010), an increase in CPN60 allowed

427 the protein to fold correctly, thereby enabling it to perform its role in the preservation and

428 modification of Rubisco subunits (Demirevska-Kepova \& Feller 2004; Gammulla et al. 2010).

429 Hsp70 and CPN60 in chloroplasts may sequentially assist in the maturation of newly imported

430 ferredoxin-NADP reductase in an ATP-dependent manner (Tsugeki \& Nishimura 1993). The

431 chloroplast genes encoding ferredoxin-NADP reductase (MH06g0016000), cytochrome b6-f

432 complex iron-sulfur subunit (MH07g0421500), and PsbP-like protein 2 (MH03g0175000) were

433 significantly down-regulation after $24 \mathrm{~h}$ of heat stress and then reverted back to control levels at

$43472 \mathrm{~h}$. The transcript levels of these genes were higher in II8 than in the parental lines, which

435 indicated they may play key roles in repairing the damage caused by photo-oxidation after long-

436 term heat treatment (Cramer 2019; Higuchi-Takeuchi et al. 2011; Pawel et al. 2012). The

437 chlorophyll content in leaves of creeping bentgrass was found to increase under heat stress (Liu

438 \& Huang 2000). Our previous data showed that the chlorophyll content of II8 was higher than it 
439

440

441

442

was in the parental lines after heat-stress exposure, and chlorophyll content significant increased after $24 \mathrm{~h}$ of heat stress (Wang et al. 2016b). Compared with the parental lines, the hybrid can maintain relatively high photosynthetic protein activity levels under the same heat stress conditions. Therefore, these over-dominant genes in the photosynthesis pathway may be related to the higher heat tolerance of the hybrid compared with its parents.

The activity of antioxidant enzymes showed positive correlation with chlorophyll concentration in wheat plant cells under high temperature stress (Almeselmani et al. 2006). Genes with a role in antioxidant defense systems, such as the peroxidases (MH04g0704000, MH07g0536400, MH05g0063600), were positively expressed in II8 under heat stress. The transcript levels of peroxidase 12 (MH04g0704000) in II8 were continuously up-regulated and were higher than those of the parental lines under the same heat-stress conditions. Consistent with our previous study, the activity levels of antioxidant enzymes (peroxidase, superoxide dismutase, catalase) in the hybrid were higher than those in the paternal lines under heat stress conditions (Wang et al. 2016b). The hybrid was more efficient than the parental lines in scavenging ROS because it had elevated antioxidant transcript and protein levels. The elevated activity of antioxidant enzymes in the hybrid may facilitate the reestablishment of cellular homeostasis to confer resistance to heat stress.

The abundance of calmodulin-binding transcription activator 2 (MH03g0091100) was increased in the hybrid as assessed by the proteomic analysis. Calmodulin is involved in Hsp gene expression through the regulation of HSF activity (Li et al. 2004; Sarkar et al. 2014). Heat stress also activates MAPKs, which are important for Hsp gene expression and thermotolerance 
460 (Sarkar et al. 2014). The transcript level of MAP3K (MH01g0546600) in II8 was continuously 461 up-regulated and was higher than its levels in the parental lines. MAP3K is responsible for 462 MAPK activation in MAPK-signaling pathways related to abiotic stress. MAP3K genes also are 463 up-regulated by heat stress, drought, $\mathrm{H}_{2} \mathrm{O}_{2}$, salt, and cold stresses (Mittal et al. 2012; Sarkar et al. 464 2014). Thus, calmodulin and protein kinases play significant roles in signaling during heat stress. Members of the HSF-Hsp network had over-dominant expression patterns in the hybrid rice compared with their expression patterns in its parents, which may help maintain relatively higher activity levels in photosynthesis and antioxidant defense systems (Fig. 7; Fig. S3). These genes were non-additively expressed under long-term heat stress in the heat tolerance co-regulatory network. The over-dominant mode of inheritance is important to heterosis because a single gene can potentially create the heterotic effect (Shapira \& David 2016). Non-additively expressed genes are candidates for contributing to heterosis (Fujimoto et al. 2018). These significantly differentially expressed over-dominant genes and non-additive genes may contribute synergistically to heterosis. These genes in the HSF-Hsp network maintained the higher activity of photosynthesis and antioxidant defense systems, that confer the hybrid rice more heat tolerance compared to the parental lines. These genes may contribute to the stronger heat resistance of the hybrid by using a different control mechanism. Thus, the findings of this study will help to advance the understanding of the heterosis of heat stress tolerance in rice.

\section{Conclusions}

The genome-wide transcriptome and proteome analyses of cultured hybrid rice II8 provides an 
481

482

483

484

485

486

487

488

489

490

491

492

493

494

495

496

497

498

499

500

501

502

503

504

505

506

507

508

unbiased methodology to investigate expression patterns of genes and abundances of proteins

using digital signals. It is evident that the heat tolerance of hybrid rice is associated with a

complicated regulatory network. In the HSF-Hsp network, the over-dominant expressed genes

may help to maintain the relatively higher activity levels of Hsps, photosynthesis, and

antioxidant defense systems that contribute to the heat tolerance of the hybrid rice. Our results

provide insights into the heat tolerance mechanisms of hybrid rice and could be applied to

develop new strategies for improving heat resistance in rice.

\section{Acknowledgements}

We thank Dr. Wei Li from the University of Michigan for critically reading the manuscript. We thank Lesley Benyon, $\mathrm{PhD}$, and Margaret Biswas, $\mathrm{PhD}$, from Liwen Bianji, Edanz Group China (www.liwenbianji.cn/ac), for editing the English text of drafts of this manuscript.

\section{References}

Almeselmani M, Deshmukh PS, Sairam RK, Kushwaha SR, and Singh TP. 2006. Protective role of antioxidant enzymes under high temperature stress. Plant Sci 171:382-388. 10.1016/j.plantsci.2006.04.009

Anjum SA, Tanveer M, Ashraf U, Hussain S, Shahzad B, Khan I, and Wang L. 2016. Effect of progressive drought stress on growth, leaf gas exchange, and antioxidant production in two maize cultivars. Environ Sci Pollut Res Int 23:17132-17141. 10.1007/s11356-0166894-8

Awad W, Estrada I, Shen Y, and Hendershot LM. 2008. BiP Mutants That Are Unable to Interact with Endoplasmic Reticulum DnaJ Proteins Provide Insights into Interdomain Interactions in BiP. Proceedings of the National Academy of Sciences of the United States of America 105:1164-1169.

Baryshnikova A. 2016. Exploratory Analysis of Biological Networks through Visualization, Clustering, and Functional Annotation in Cytoscape. Cold Spring Harb Protoc 2016. 10.1101/pdb.prot077644 
509 Cao YY, Duan H, Yang LN, Wang ZQ, Zhou SC, and Yang JC. 2008. Effect of Heat Stress

510

511

512

513

514

515

516

517

518

519

520

521

522

523

524

525

526

527

528

529

530

531

532

533

534

535

536

537

538

539

540

541

542

543

544

545

546

547

548

549 During Meiosis on Grain Yield of Rice Cultivars Differing in Heat Tolerance and Its Physiological Mechanism. Acta Agronomica Sinica 34:2134-2142.

Cheabu S, Moung-Ngam P, Arikit S, Vanavichit A, and Malumpong C. 2018. Effects of Heat Stress at Vegetative and Reproductive Stages on Spikelet Fertility. Rice Science 25:218226.

Cramer WA. 2019. Structure-function of the cytochrome b6f lipoprotein complex: a scientific odyssey and personal perspective. Photosynth Res 139:53-65. 10.1007/s11120-018-0585$\mathrm{x}$

Damaris RN, Ming L, Liu Y, Chen X, Murage H, and Yang P. 2016. A proteomic analysis of salt stress response in seedlings of two African rice cultivars. BBA - Proteins and Proteomics 1864:1570-1578.

Demirevska-Kepova K, and Feller U. 2004. Heat sensitivity of Rubisco, Rubisco activase and Rubisco binding protein in higher plants. Acta Physiologiae Plantarum 26:103-114.

Deng D, Chen H, Deng W, Feng L, Yang X, Ma A, and Liu M. 2009. Development of a Indica rice restorer line FUHUI838 and its derivative lines with strong restoring ability and their utilization. Journal of Nuclear Agricultural Sciences 23:175-179.

Dias A, Semedo J, Ramalho J, and Lidon F. 2011. Bread and durum wheat under heat stress: a comparative study on the photosynthetic performance. Journal of Agronomy and Crop Science 197:50-56.

Feng SQ, Chen XL, Wu SJ, and Chen XS. 2015. Recent advances in understanding plant heterosis. Agricultural Sciences 06:1033-1038.

Fujimoto R, Uezono K, Ishikura S, Osabe K, Peacock WJ, and Dennis ES. 2018. Recent research on the mechanism of heterosis is important for crop and vegetable breeding systems. Breeding Science 68:145-158.

Gammulla CG, Pascovici D, Atwell BJ, and Haynes PA. 2010. Differential metabolic response of cultured rice (Oryza sativa) cells exposed to high- and low-temperature stress. Proteomics 10:3001-3019.

Hahn A, and Scharf KD. 2011. Crosstalk between Hsp90 and Hsp70 chaperones and heat stress transcription factors in tomato. Plant Cell 23:741-755.

Han R, Ruqi SU, Wan J, Long Q, Zeng Y, Pan X, Shi Q, and Ziming WU. 2018. Protective Roles of Over-expression of OsXDH in Rice Seedlings Under High Temperature Stress. Chinese Journal of Rice Science.

Hasanuzzaman M, Nahar K, Alam MM, Roychowdhury R, and Fujita M. 2013. Physiological, biochemical, and molecular mechanisms of heat stress tolerance in plants. Int J Mol Sci 14:9643-9684. 10.3390/ijms14059643

Higuchi-Takeuchi M, Ichikawa T, Kondou Y, Matsui K, Hasegawa Y, Kawashima M, Sonoike K, Mori M, Hirochika H, and Matsui M. 2011. Functional analysis of two isoforms of leaf-type ferredoxin-NADP(+)-oxidoreductase in rice using the heterologous expression system of Arabidopsis. Plant Physiol 157:96-108. 10.1104/pp.111.181248

Hochholdinger F, and Baldauf JA. 2018. Heterosis in plants. Curr Biol 28:R1089-R1092. 
550

551

552

553

554

555

556

557

558

559

560

561

562

563

564

565

566

567

568

569

570

571

572

573

574

575

576

577

578

579

580

581

582

583

584

585

586

587

588

589

590

10.1016/j.cub.2018.06.041

Jain M, Nijhawan A, Tyagi AK, and Khurana JP. 2006. Validation of housekeeping genes as internal control for studying gene expression in rice by quantitative real-time PCR. Biochemical and biophysical research communications 345:646-651.

Jiang Y, Chen C, Tao X, Wang J, and Zhang Y. 2012. A proteomic analysis of storage stress responses in Ipomoea batatas (L.) Lam. tuberous root. Molecular Biology Reports 39:8015-8025. 10.1007/s11033-012-1648-2

Kim JS, Yamaguchi-Shinozaki K, and Shinozaki K. 2018. ER-Anchored Transcription Factors bZIP17 and bZIP28 Regulate Root Elongation. Plant Physiol 176:2221-2230. 10.1104/pp.17.01414

Li B, Gao K, Ren H, and Tang W. 2018a. Molecular mechanisms governing plant responses to high temperatures. J Integr Plant Biol 60:757-779. 10.1111/jipb.12701

Li B, Liu HT, Sun DY, and Zhou RG. 2004. Ca(2+) and calmodulin modulate DNA-binding activity of maize heat shock transcription factor in vitro. Plant Cell Physiol 45:627-634. $10.1093 / \mathrm{pcp} / \mathrm{pch} 074$

Li J, and Liu X. 2019. Genome-wide identification and expression profile analysis of the Hsp20 gene family in Barley (Hordeum vulgare L.). PeerJ 7:e6832. 10.7717/peerj.6832

Li MM, Li X, Yu LQ, Wu JW, Li H, Liu J, Ma XD, Jo SM, Park DS, and Song Y. 2018b. Identification of QTLs associated with heat tolerance at the heading and flowering stage in rice ( Oryza sativa L.). Euphytica 214:70.

Li S, Fu Q, Chen L, Huang W, and Yu D. 2011. Arabidopsis thaliana WRKY25, WRKY26, and WRKY33 coordinate induction of plant thermotolerance. Planta 233:1237-1252. $10.1007 / \mathrm{s} 00425-011-1375-2$

Liu X, and Huang B. 2000. Heat stress injury in relation to membrane lipid peroxidation in creeping bentgrass. Crop Science 40:503-510.

Liu Z, Wang P, Zhang T, Li Y, Wang Y, and Gao C. 2018. Comprehensive analysis of BpHSP genes and their expression under heat stresses in Betula platyphylla. Environmental \& Experimental Botany.

Lu SJ, Yang ZT, Sun L, Sun L, Song ZT, and Liu JX. 2012. Conservation of IRE1-regulated bZIP74 mRNA unconventional splicing in rice (Oryza sativa L.) involved in ER stress responses. Molecular Plant 5:504-514. 10.1093/mp/ssr115

Mittal D, Madhyastha DA, and Grover A. 2012. Genome-wide transcriptional profiles during temperature and oxidative stress reveal coordinated expression patterns and overlapping regulons in rice. PloS one 7:e40899.

Pawel B, Wilson KE, and Gray GR. 2012. The PSBP2 protein of Chlamydomonas reinhardtii is required for singlet oxygen-dependent signaling. Planta 236:1289-1303.

Peng S, Huang J, Sheehy JE, Laza RC, Visperas RM, Zhong X, Centeno GS, Khush GS, and Cassman KG. 2004. Rice yields decline with higher night temperature from global warming. Proceedings of the National Academy of Sciences of the United States of America 101:9971-9975.

Poli Y, Basava RK, Panigrahy M, Vinukonda VP, Dokula NR, Voleti SR, Desiraju S, and 
591

592

593

594

595

596

597

598

599

600

601

602

603

604

605

606

607

608

609

610

611

612

613

614

615

616

617

618

619

620

621

622

623

624

625

626

627

628

629

630

631

Neelamraju S. 2013. Characterization of a Nagina22 rice mutant for heat tolerance and mapping of yield traits. Rice 6:36.

Sarkar NK, Kim YK, and Grover A. 2014. Coexpression network analysis associated with call of rice seedlings for encountering heat stress. Plant Mol Biol 84:125-143. 10.1007/s11103013-0123-3

Scafaro AP, Haynes PA, and Atwell BJ. 2010. Physiological and molecular changes inOryza meridionalisNg., a heat-tolerant species of wild rice. Journal of experimental botany 61:191-202.

Shapira R, and David L. 2016. Genes with a Combination of Over-Dominant and Epistatic Effects Underlie Heterosis in Growth ofSaccharomyces cerevisiaeat High Temperature. Frontiers in Genetics 7.

Sinha P, Poland J, Schnolzer M, and Rabilloud T. 2001. A new silver staining apparatus and procedure for matrix-assisted laser desorption/ionization-time of flight analysis of proteins after two-dimensional electrophoresis. Proteomics 1:835-840. 10.1002/16159861(200107)1:7<835::AID-PROT835>3.0.CO;2-2

Song JM, Lei Y, Shu CC, Ding Y, Xing F, Liu H, Wang J, Xie W, Zhang J, and Chen LL. 2018. Rice Information GateWay: A Comprehensive Bioinformatics Platform for Indica Rice Genomes. Molecular Plant 11:505-507. 10.1016/j.molp.2017.10.003

Su P, Feng H, Chen H, Yang C, Wu X, and Huang Q. 2013. Influences of Natural Hightemperature Stress on Heat Resistance of Rice. Southwest China Journal of Agricultural Sciences 26:1387-1391.

Szklarczyk D, Morris JH, Cook H, Kuhn M, Wyder S, Simonovic M, Santos A, Doncheva NT, Roth A, Bork P, Jensen LJ, and von Mering C. 2017. The STRING database in 2017: quality-controlled protein-protein association networks, made broadly accessible. Nucleic Acids Res 45:D362-D368. 10.1093/nar/gkw937

Takahashi H, Kawakatsu T, Wakasa Y, Hayashi S, and Takaiwa F. 2012. A rice transmembrane bZIP transcription factor, OsbZIP39, regulates the endoplasmic reticulum stress response. Plant Cell Physiol 53:144-153. 10.1093/pcp/pcr157

Tao X, Gu YH, Wang HY, Zheng W, Li X, Zhao CW, and Zhang YZ. 2012. Digital Gene Expression Analysis Based on Integrated De Novo Transcriptome Assembly of Sweet Potato [Ipomoea batatas (L.) Lam.]. PloS one 7:e36234.

Tian T, Liu Y, Yan H, You Q, Yi X, Du Z, Xu W, and Su Z. 2017. agriGO v2.0: a GO analysis toolkit for the agricultural community, 2017 update. Nucleic acids research 45:W122W129.

Trapnell C, Williams BA, Pertea G, Mortazavi A, Kwan G, van Baren MJ, Salzberg SL, Wold BJ, and Pachter L. 2010. Transcript assembly and quantification by RNA-Seq reveals unannotated transcripts and isoform switching during cell differentiation. Nat Biotechnol 28:511-515. 10.1038/nbt.1621

Tsugeki R, and Nishimura M. 1993. Interaction of homologues of Hsp70 and Cpn60 with ferredoxin-NADP + reductase upon its import into chloroplasts. FEBS letters 320:198.

Vandesompele J, De Preter K, Pattyn F, Poppe B, Van Roy N, De Paepe A, and Speleman F. 
632

633

634

635

636

637

638

639

640

641

642

643

644

645

646

647

648

649

650

651

652

653

654

655

656

657

658

659

660

661

662

663

664

665

666

667

668

669

670

671

672

2002. Accurate normalization of real-time quantitative RT-PCR data by geometric averaging of multiple internal control genes. Genome Biol 3:RESEARCH0034.

Wang D, Qin B, Li X, Tang D, Zhang Y, Cheng Z, and Xue Y. 2016a. Nucleolar DEAD-Box RNA Helicase TOGR1 Regulates Thermotolerant Growth as a Pre-rRNA Chaperone in Rice. Plos Genetics 12:e1005844.

Wang Y, Gao P, Fu Y, He J, Sun Q, and Huang M. 2016b. Identification of Heat Resistance in Radiation Breed Hybrid Rice II YOU 838. Hubei Agricultural Sciences:6061-6066.

Wang Y, Wang L, Zhou J, Hu S, Chen H, Xiang J, Zhang Y, Zeng Y, Shi Q, Zhu D, and Zhang Y. 2019. Research Progress on Heat Stress of Rice at Flowering Stage. Rice Science 26:1-10.

Wei G, Tao Y, Liu G, Chen C, Luo R, Xia H, Gan Q, Zeng H, Lu Z, and Han Y. 2009. A transcriptomic analysis of superhybrid rice LYP9 and its parents. Proceedings of the National Academy of Sciences 106:7695.

Wilson RH, and Hayer-Hartl M. 2018. Complex Chaperone Dependence of Rubisco Biogenesis. Biochemistry 57:3210-3216. 10.1021/acs.biochem.8b00132

Xing X, Li X, Zhang M, Wang Y, Liu B, Xi Q, Zhao K, Wu Y, and Yang T. 2017. Transcriptome analysis of resistant and susceptible tobacco (Nicotiana tabacum) in response to root-knot nematode Meloidogyne incognita infection. Biochem Biophys Res Commun 482:1114-1121. 10.1016/j.bbrc.2016.11.167

Ye J, Coulouris G, Zaretskaya I, Cutcutache I, Rozen S, and Madden TL. 2012. Primer-BLAST: a tool to design target-specific primers for polymerase chain reaction. $B M C$ Bioinformatics 13:134. 10.1186/1471-2105-13-134

Zafar SA, Hameed A, Nawaz MA, Wei MA, Noor MA, Hussain M, and Mehboob-ur-Rahman. 2018. Mechanisms and molecular approaches for heat tolerance in rice ( Oryza sativa L.) under climate change scenario. Journal of Integrative Agriculture 17:726-738.

Zhang C, Li G, Chen T, Feng B, Fu W, Yan J, Islam MR, Jin Q, Tao L, and Fu G. 2018. Heat stress induces spikelet sterility in rice at anthesis through inhibition of pollen tube elongation interfering with auxin homeostasis in pollinated pistils. Rice 11:14.

Zhang J, Chen LL, Shuai S, Kudrna D, Copetti D, Li W, Mu T, Jiao WB, Feng X, and Lee S. 2016a. Building two indica rice reference genomes with PacBio long-read and Illumina paired-end sequencing data. Scientific Data 3:160076.

Zhang J, Chen LL, Xing F, Kudrna DA, Yao W, Copetti D, Mu T, Li W, Song JM, and Xie W. 2016b. Extensive sequence divergence between the reference genomes of two elite indica rice varieties Zhenshan 97 and Minghui 63. Proc Natl Acad Sci U S A 113:E5163.

Zhang JJ, Zhou ZS, Song JB, Liu ZP, and Yang H. 2012a. Molecular dissection of atrazineresponsive transcriptome and gene networks in rice by high-throughput sequencing. Journal of hazardous materials.

Zhang X, Li J, Liu A, Zou J, Zhou X, Xiang J, Rerksiri W, Peng Y, Xiong X, and Chen X. 2012b. Expression profile in rice panicle: insights into heat response mechanism at reproductive stage. PloS one 7:e49652. 10.1371/journal.pone.0049652

Zhang X, Rerksiri W, Liu A, Zhou X, Xiong H, Xiang J, Chen X, and Xiong X. 2013. 
673 Transcriptome profile reveals heat response mechanism at molecular and metabolic levels $674 \quad$ in rice flag leaf. Gene 530:185-192. 10.1016/j.gene.2013.08.048

675 Zhao Q, Zhou L, Liu J, Du X, Asad MA, Huang F, Pan G, and Cheng F. 2018. Relationship of 676 ROS accumulation and superoxide dismutase isozymes in developing anther with floret 677 fertility of rice under heat stress. Plant Physiol Biochem 122:90-101.

$678 \quad$ 10.1016/j.plaphy.2017.11.009

679 Zhu JK. 2016. Abiotic Stress Signaling and Responses in Plants. Cell 167:313-324.

$680 \quad 10.1016 /$ j.cell.2016.08.029

681 Zou J, Liu C, and Chen X. 2011. Proteomics of rice in response to heat stress and advances in 682 genetic engineering for heat tolerance in rice. Plant cell reports 30:2155-2165.

683 


\section{Figure 1}

Changes in flag leaves and gene expression between hybrid rice (II8) and the paternal F8 and maternal II3 lines after heat stress.

(A) Flag leaves of 118 and the parental lines after $120 \mathrm{~h}$ of heat stress at $42^{\circ} \mathrm{C}$. CK, unheated

$(0 \mathrm{~h})$ control group; heat treatment, heat treated for $120 \mathrm{~h}$ group. (B) Numbers of the differentially expressed genes between II8 and the parental lines after different durations of heat stress. II8, hybrid rice II YOU 838; F8, paternal Fu Hui 838; II3, maternal II-32A. 
A

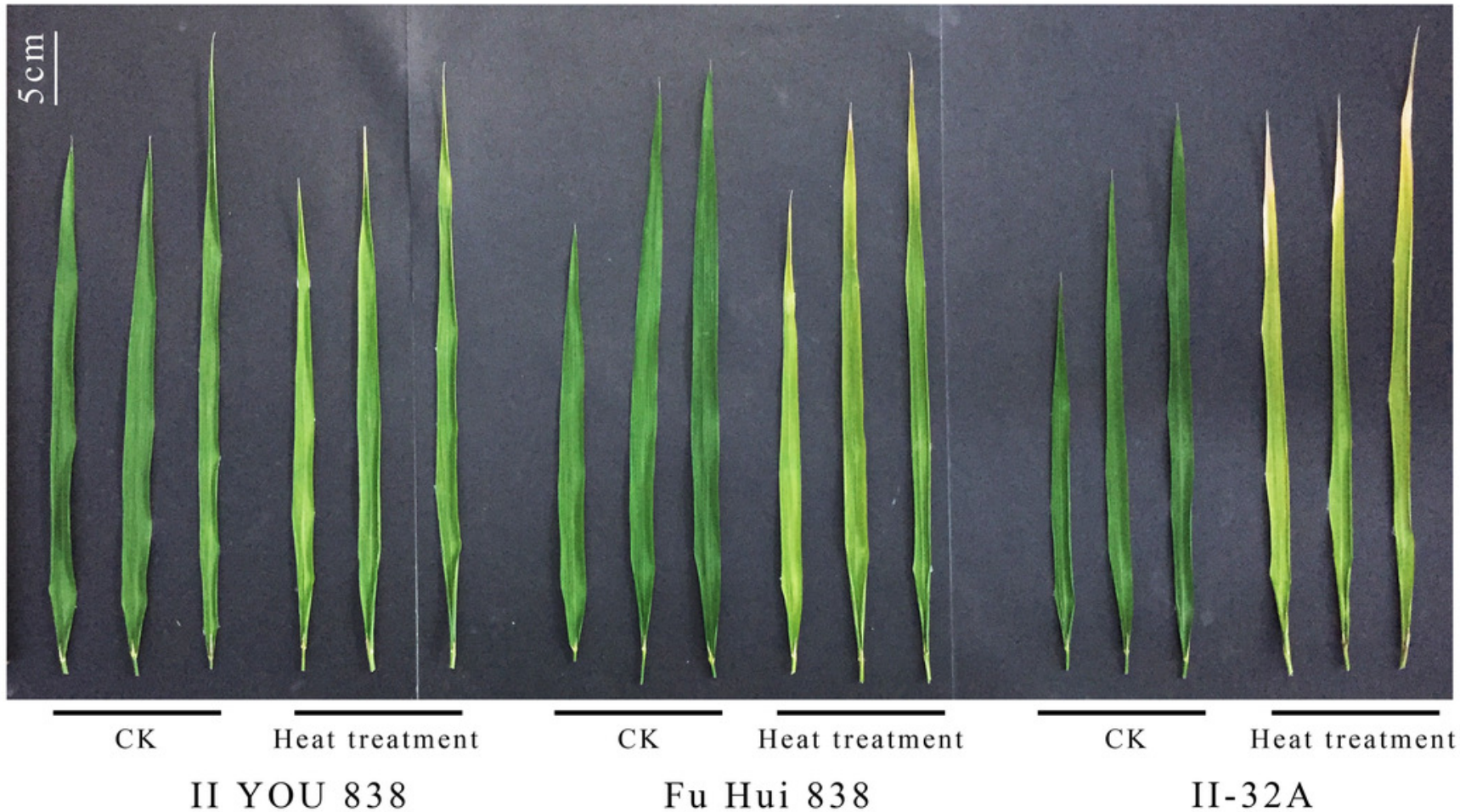

B

$0 \mathrm{~h}$ vs $24 \mathrm{~h}$

$0 \mathrm{~h}$ vs $72 \mathrm{~h}$
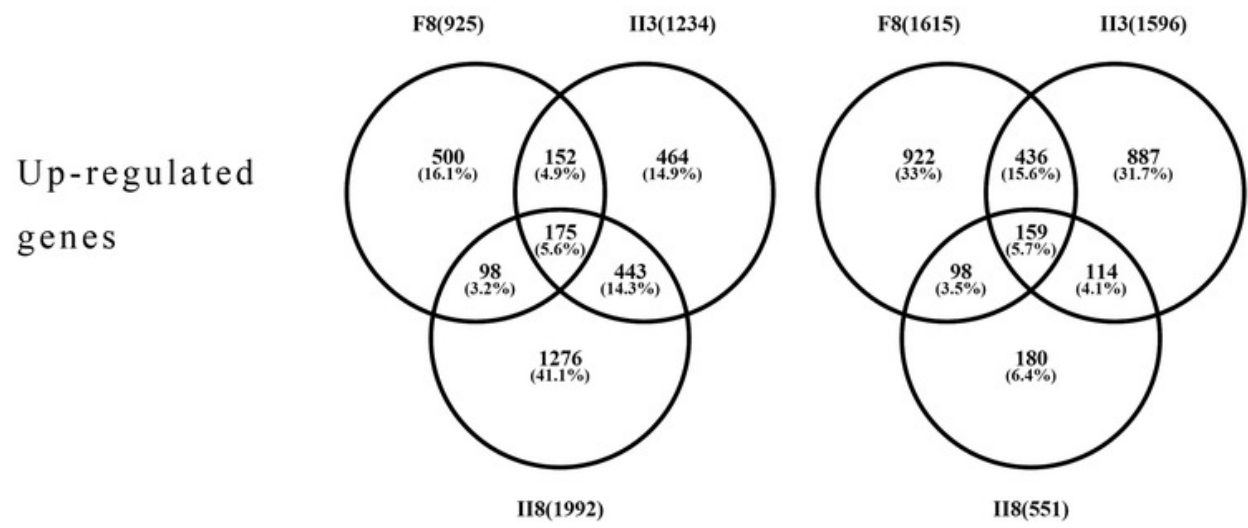

II8(551)
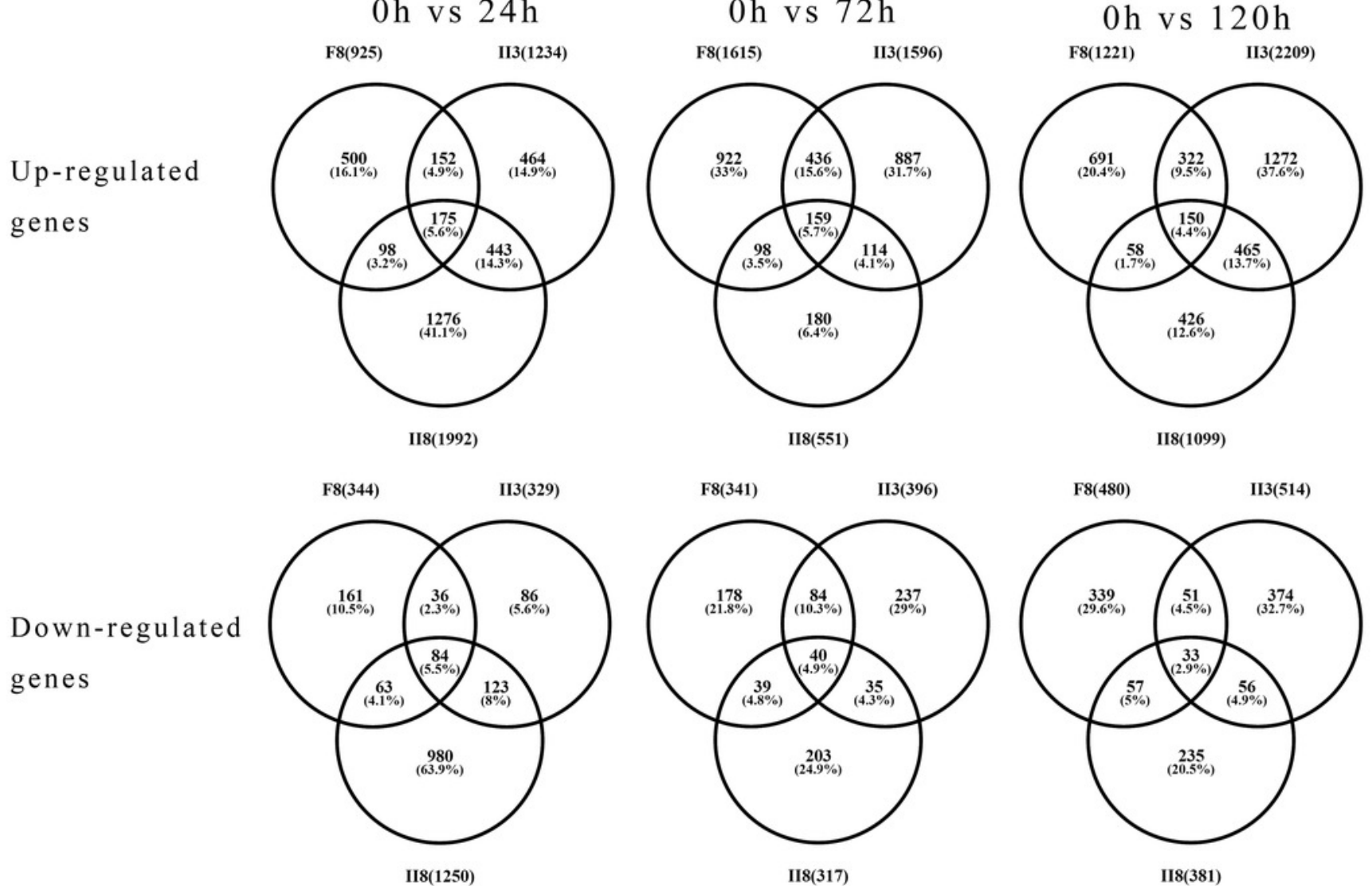

II8(1099)

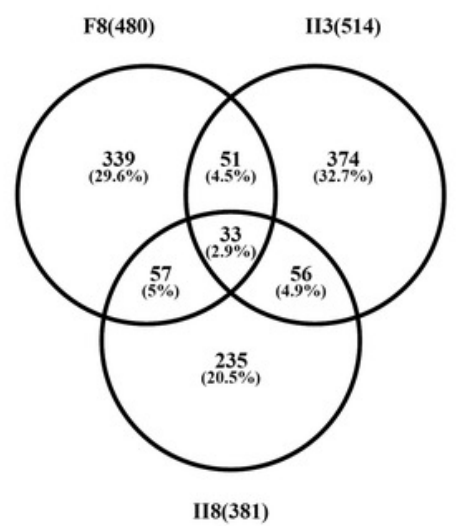




\section{Figure 2}

Differential gene expression in hybrid rice (II8) in response to different durations of heat stress.

(A) Number of differentially expressed genes (DEGs) in II8 after heat stress for 0 h, 24 h, 72 h, and $120 \mathrm{~h}$. (B) Venn diagram of the DEGs in II8 at all the time points. (C) Hierarchical clustering analysis of II8 of 586 significantly regulated DEGs at all the time points.
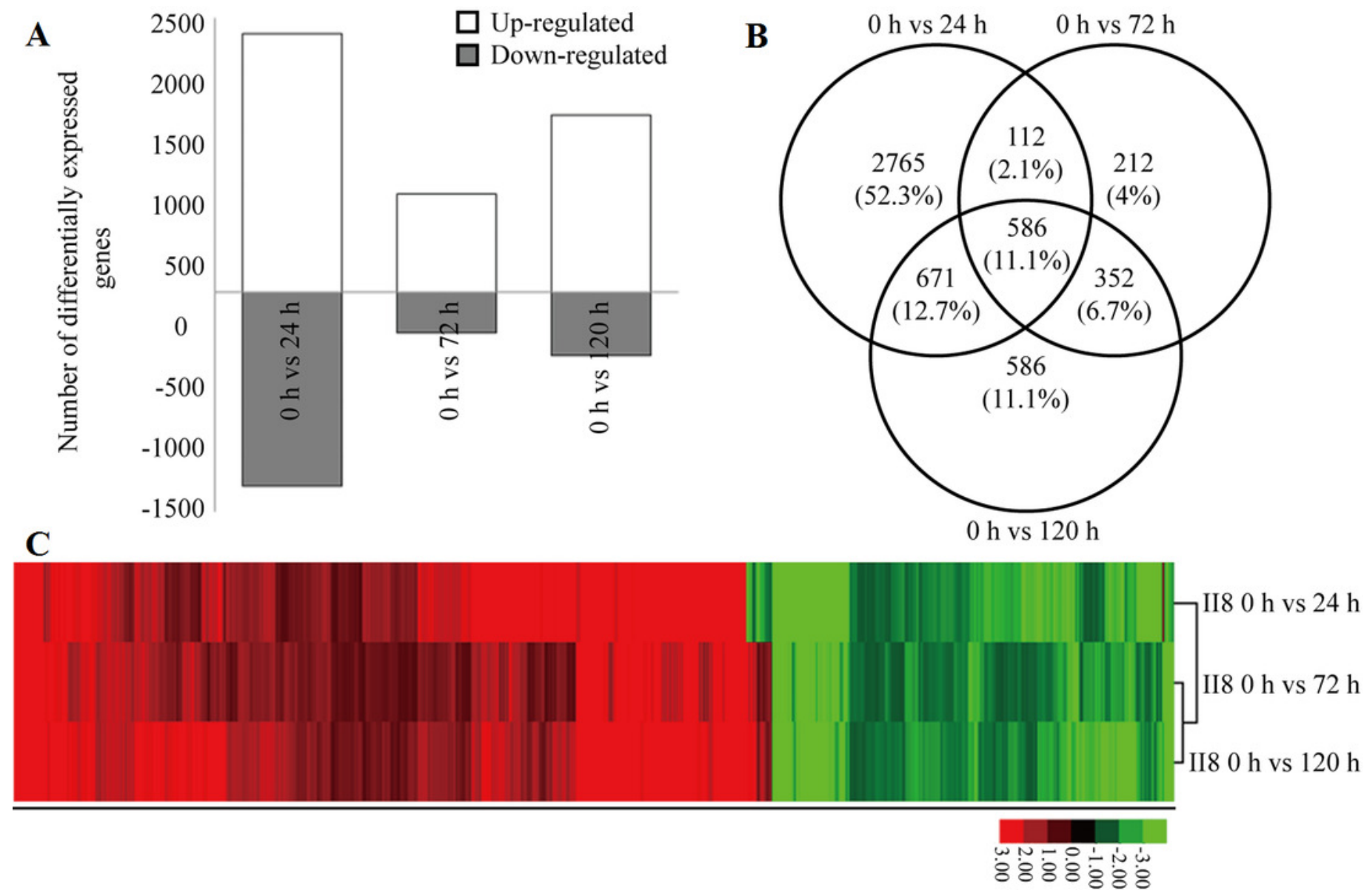


\section{Figure 3}

Expression patterns of the differentially expressed genes in hybrid rice (II8) flag leaves after different durations of heat-stress.

The vertical axis represents the gene expression signal values centered on log2 (fragments per kilobase per million +1$)$, and the horizontal axis represents the four sample times $(0 \mathrm{~h}$, 24 h, 72 h, 120 h). (A) Genes in Cluster A were continuously down-regulated. (B and C) Genes in Clusters B and C were continuously up-regulated. (D-G) Genes in Clusters D-G showed early up-regulation and then partial decrease. $(\mathrm{H}-\mathrm{J})$ Genes in Clusters $\mathrm{H}-\mathrm{J}$ showed early downregulation before reversion. 
A
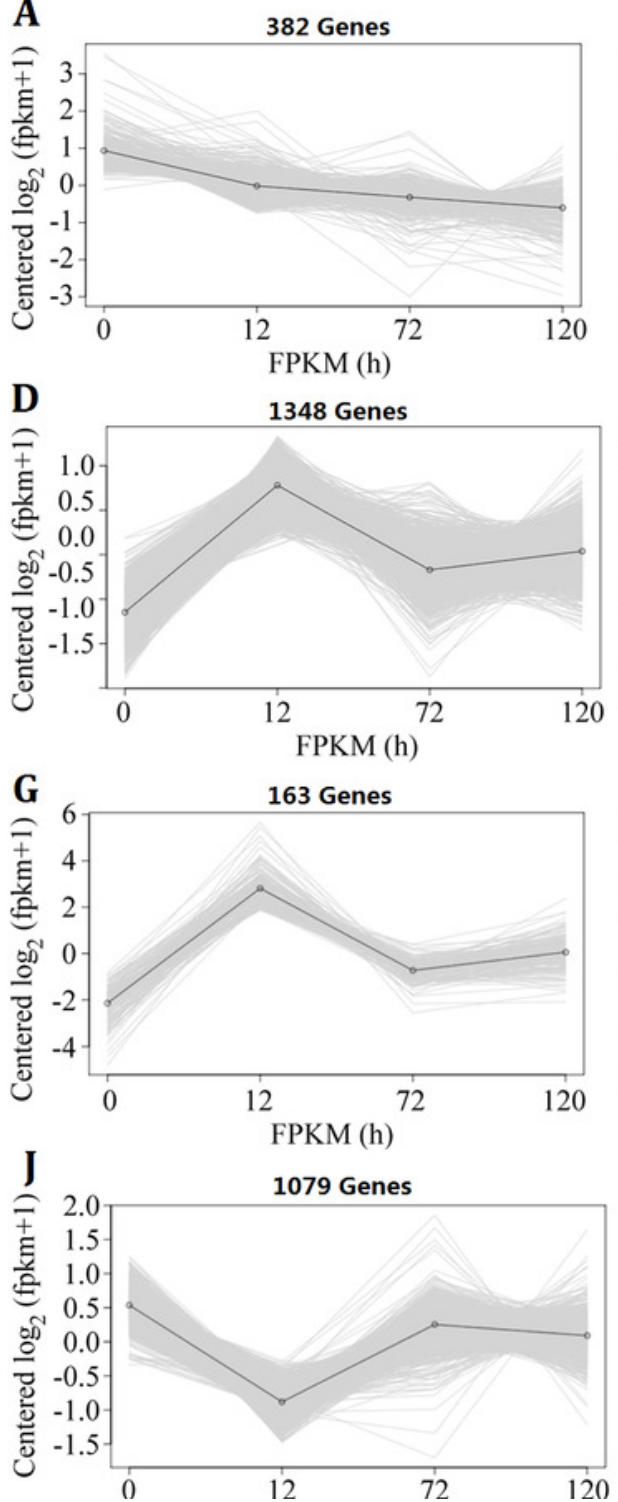

B
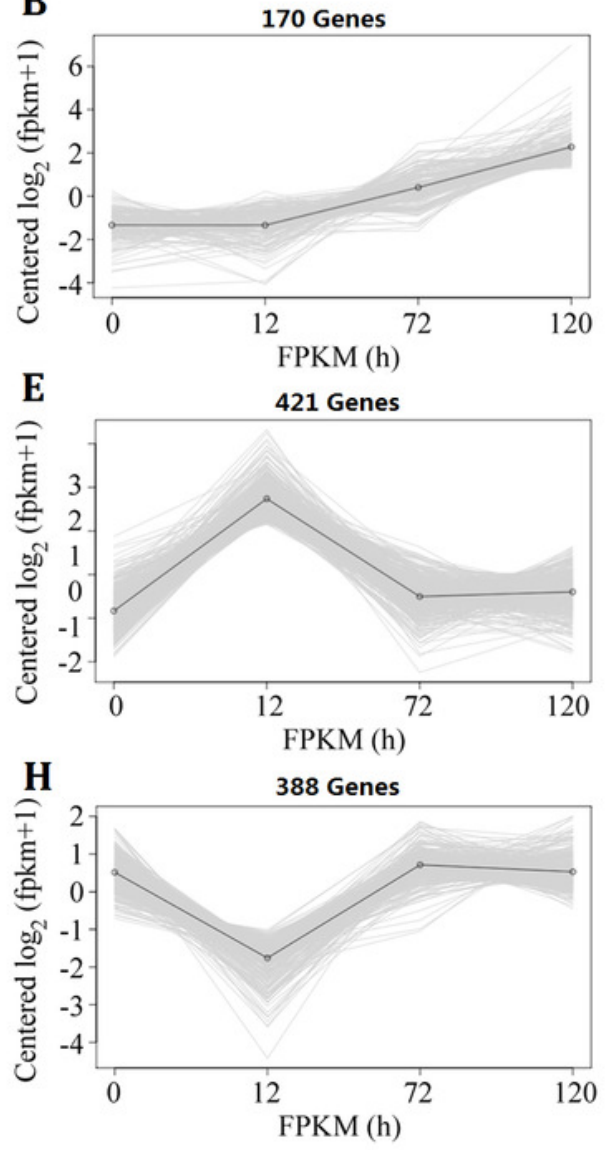
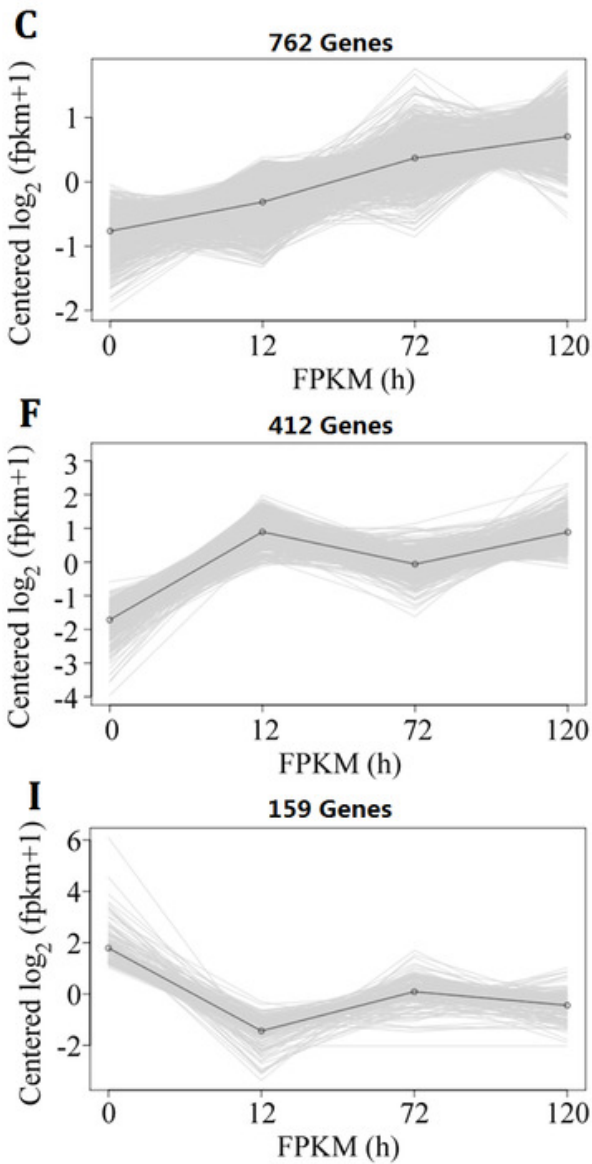
Figure 4

Gene ontology (GO) enrichment analysis of the hybrid rice (II8) genes in Clusters generated by k-means clustering.

$(A, B)$ Distribution of the GO terms assigned to genes in the early up- and down-regulated clusters. (C, D) Distribution of the GO terms assigned to genes in the continuous up- and down-regulated clusters. 


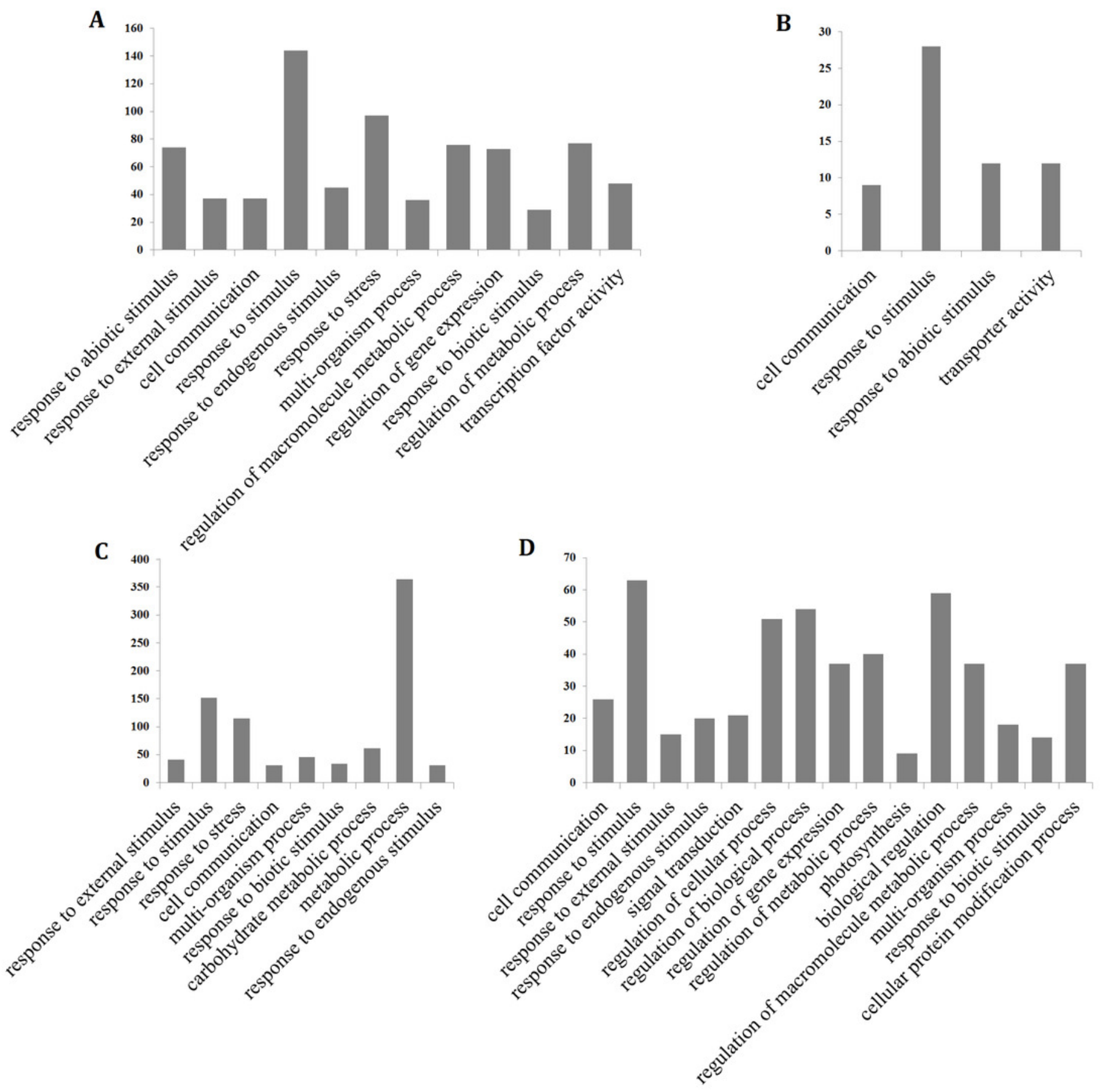




\section{Figure 5}

Quantitative real-time RT-PCR (qPCR) validation of the RNA-Seq data from II8 and the paternal F8 and maternal II3 lines.

The columns indicate the relative expression levels obtained by qPCR and the lines indicate the TPM values obtained by RNA-Seq. Error bars are standard deviations of the mean $(n=3)$ among the three repeats. The internal control genes were MH03g0618400 (ACTIN1), MH01g0139700 (Mpv17), and MH02g0177100 (chloroplast processing peptidase). The genes selected for validation are: (A) MH03g0686900, mitochondrial import inner membrane translocase subunit TIM14-3. (B) MH03g0260900, HUA2-like protein 2. (C) MH03g0018700, Hsp70. (D) MH04g0704000, peroxidase 12. (E) MH07g0536400, peroxidase 2. (F)

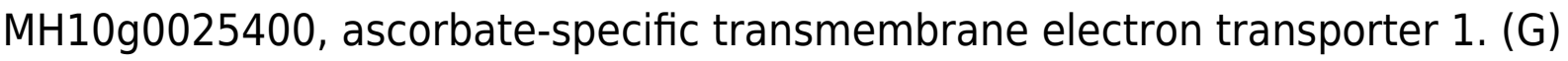
MH03g0762100, OsHsfA1. (H) MH05g0501600, HsfA4d.
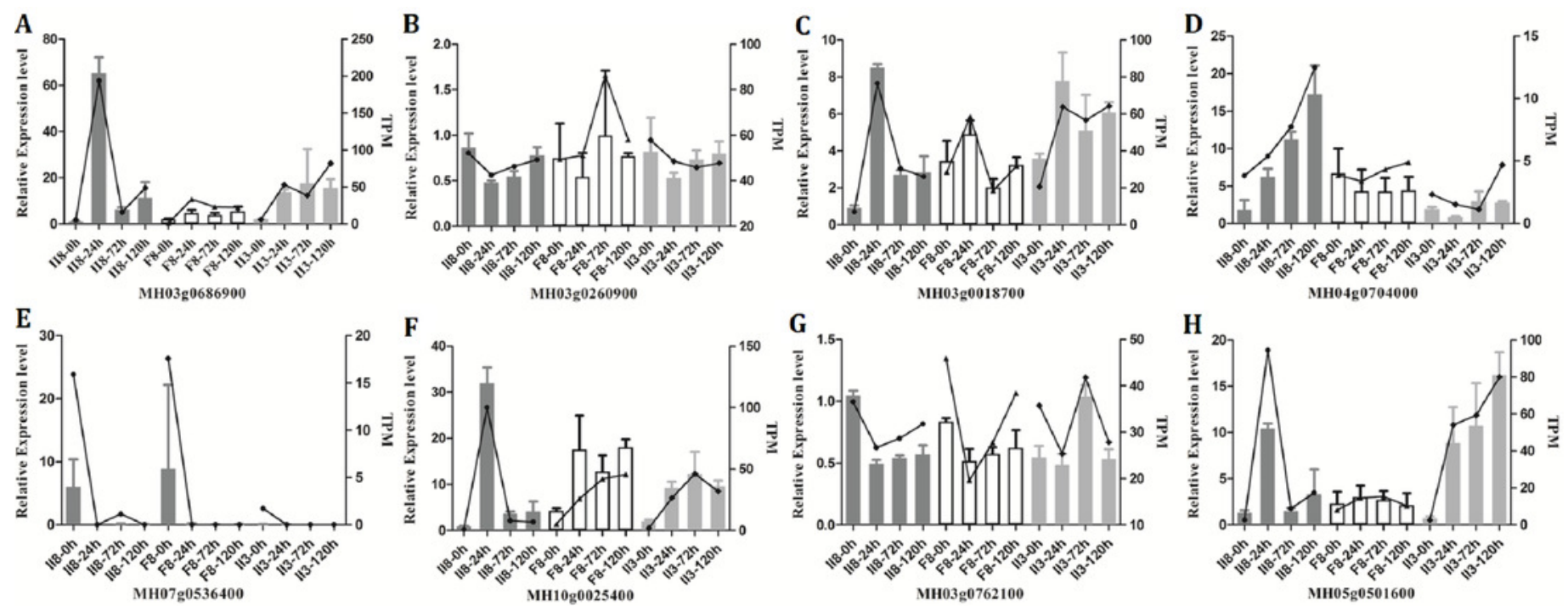


\section{Figure 6}

Co-regulatory network predicted from the differential gene and protein data of II8 flag leaves during long-term heat stress.

The protein-protein interaction networks were identified using the STRING database. It contained four subclusters; namely, cluster A, cluster B, cluster C, and cluster D. Members of the HSF-Hsp network were dark green nodes in cluster D.

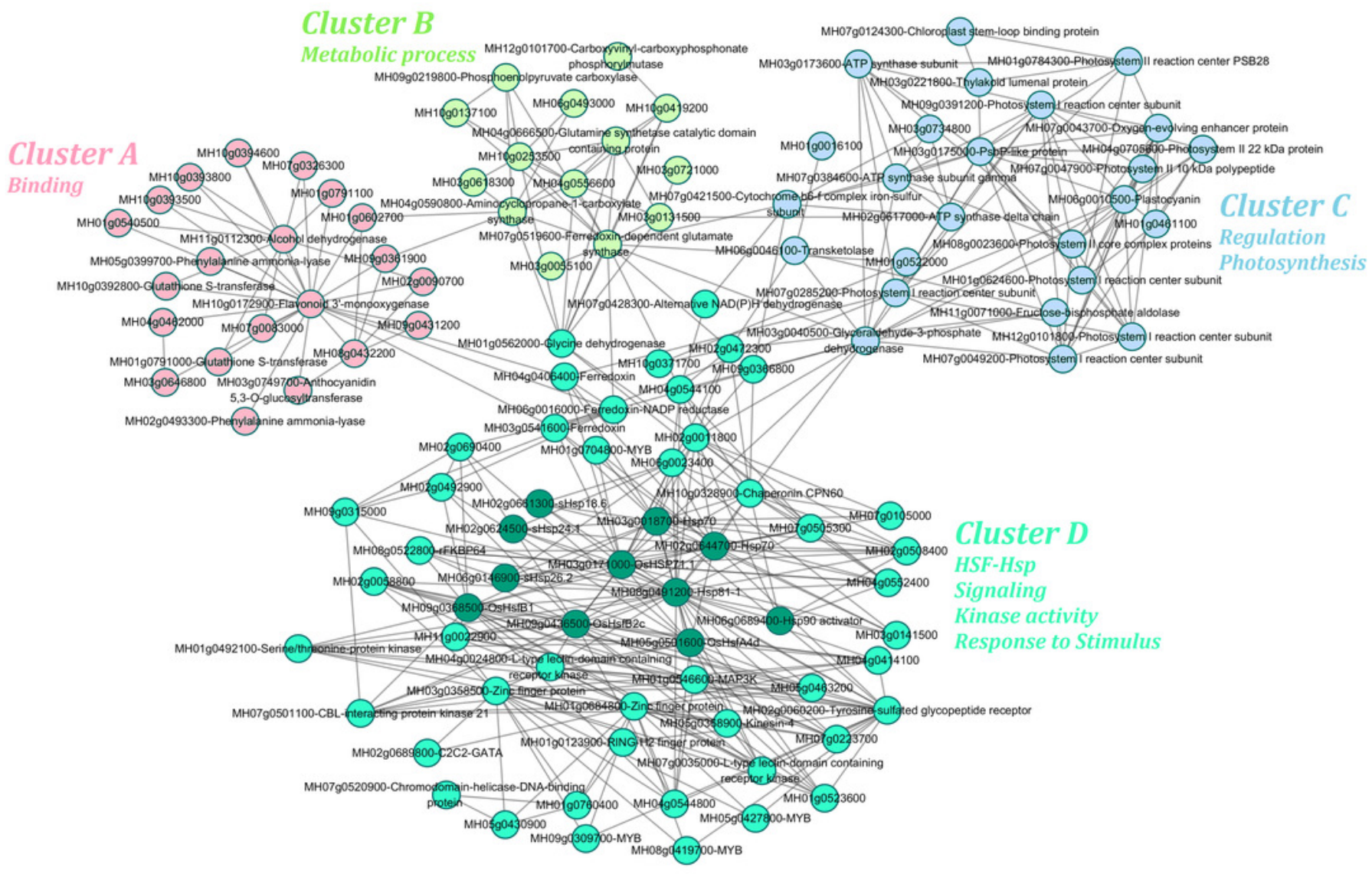




\title{
Figure 7
}

Schematic illustration of heat tolerance mechanism in the hybrid rice (II8).

\section{High temperature}

\author{
$\downarrow$ Perceive the heat stress stimulus
}

Disruption of membrane structure and cellular homeostasis

$$
\downarrow \text { Signal sensing and transduction }
$$

Triggered expression of heat shock genes

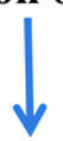

Synergistic contribution of over-dominant genes to heterosis in HSF-Hsp network

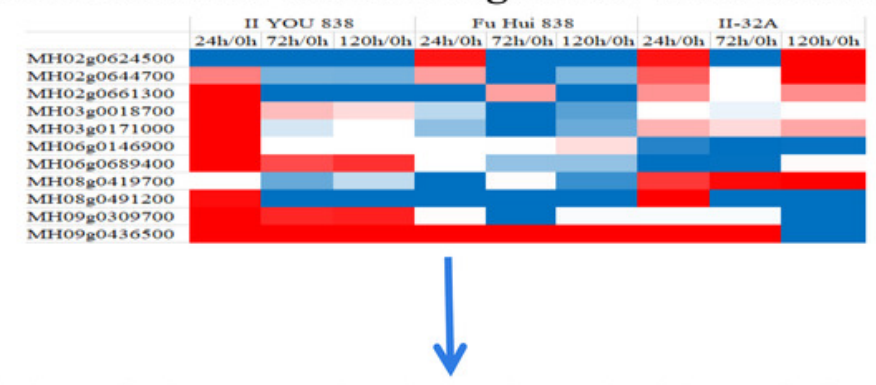

Higher activity of photosynthesis and antioxidant defense in hybrid

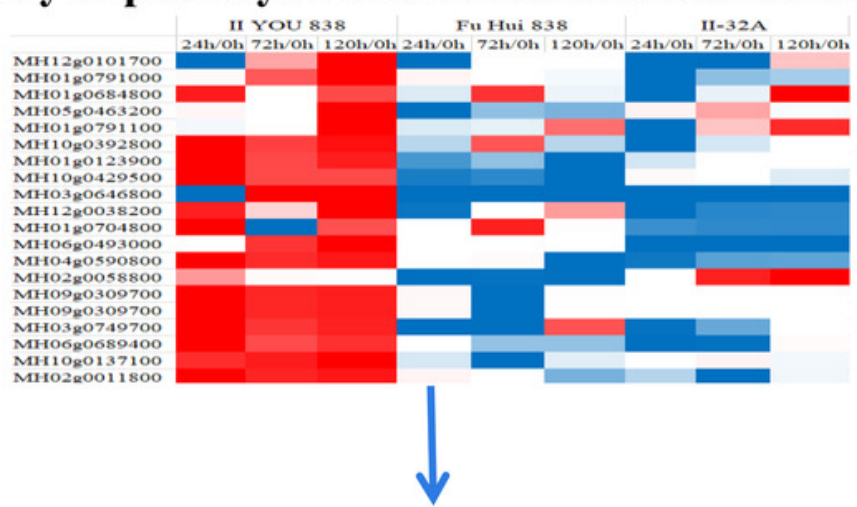

Reestablished cellular homeostasis

Development of heat tolerance in hybrid rice 


\section{Table $\mathbf{1}$ (on next page)}

The 35 unique differentially abundant proteins detected in the flag leaves of hybrid rice II YOU 838 after $24 \mathrm{~h}$ of heat stress.

a Spot numbers correspond to the protein spot numbers in Fig. S2. "+" Proteins that showed increased abundance after $24 \mathrm{~h}$ of heat stress. * The 18 proteins that showed the same abundance patterns (increased or decreased) as the corresponding gene expression levels (up- or down-regulated). 
1 Table 1: The 35 unique differentially abundant proteins detected in the flag leaves of hybrid rice 2 II YOU 838 after $24 \mathrm{~h}$ of heat stress.

\begin{tabular}{|c|c|c|c|}
\hline Spot No. ${ }^{a}$ & Gene ID & Functional annotation & Fold change \\
\hline \multicolumn{4}{|c|}{ Response to stimulus } \\
\hline 7 & MH02g0458600 & Protein FLUORESCENT IN BLUE LIGHT & $3.74 *$ \\
\hline 9 & MH07g0043700 & Oxygen-evolving enhancer protein 2 & $2.07 *$ \\
\hline 11 & MH12g0410900 & Probable disease resistance protein, leucine Rich Repeat family & 3.37 \\
\hline 16 & MH07g0011600 & Glutamate receptor 3.4 & 2.71 \\
\hline 17 & MH02g0063200 & SNF1-related protein kinase regulatory subunit gamma-like PV42a & 7.68 \\
\hline 18 & MH09g0219800 & Phosphoenolpyruvate carboxylase 2 & 2.59 \\
\hline 21 & MH02g0644700 & Heat shock $70 \mathrm{kDa}$ protein & $+2.22 *$ \\
\hline 25 & MH11g0587400 & Putative disease resistance protein RGA1 & 8.34 \\
\hline 27 & MH04g0666500 & Glutamine synthetase, chloroplastic & $2.13^{*}$ \\
\hline 45 & MH11g0166300 & Serpin-Z2B & 2.74 \\
\hline 53 & MH08g0522800 & $\begin{array}{l}70 \mathrm{kDa} \text { peptidyl-prolyl isomerase, large rice FK506 binding protein } \\
\text { gene rFKBP64 }\end{array}$ & $+2.03^{*}$ \\
\hline \multicolumn{4}{|c|}{ Oxidation-reduction } \\
\hline 24 & MH03g0040500 & Glyceraldehyde-3-phosphate dehydrogenase GAPB & $2.04 *$ \\
\hline 52 & MH06g0582000 & Glycine dehydrogenase & +2.10 \\
\hline 54 & MH08g0509600 & Probable lipoxygenase 8 & 2.40 \\
\hline 56 & MH07g0519600 & Ferredoxin-dependent glutamate synthase & $5.08^{*}$ \\
\hline \multicolumn{4}{|c|}{ Photosynthesis } \\
\hline 1 & MH07g0421500 & Cytochrome b6-f complex iron-sulfur subunit & $3.40^{*}$ \\
\hline 2 & MH06g0706000 & ATP-dependent zinc metalloprotease FTSH 1 & $+2.08 *$ \\
\hline 6 & MH05g0279300 & Putative PPR & +3.14 \\
\hline 12 & MH07g0124300 & Chloroplast stem-loop binding protein of $41 \mathrm{kDa}$ & $3.23^{*}$ \\
\hline 15 & MH10g0328900 & Chaperonin 60-2 (CPN60-2), mitochondrial & $+2.58 *$ \\
\hline 26 & MH11g0071000 & Fructose-bisphosphate aldolase & $3.46^{*}$ \\
\hline 42 & MH06g0016000 & Ferredoxin--NADP reductase chloroplast precursor & $3.05^{*}$ \\
\hline \multicolumn{4}{|c|}{ Metabolic process } \\
\hline 4 & MH02g0060200 & Tyrosine-sulfated glycopeptide receptor 1 & 2.15 \\
\hline 5 & MH02g0406900 & Probable leucine-rich repeat receptor-like protein kinase & 2.50 \\
\hline 10 & MH04g0024800 & L-type lectin-domain containing receptor kinase IV.2 & 2.68 \\
\hline 20 & MH03g0091100 & Calmodulin-binding transcription activator 2 & +2.06 \\
\hline 40 & MH09g0379400 & Transcription factor bHLH96 & $7.48^{*}$ \\
\hline 50 & MH10g0280100 & 40S ribosomal protein $\mathrm{S} 28$ & $2.17 *$ \\
\hline 55 & MH06g0046100 & Transketolase & $2.10^{*}$ \\
\hline 3 & MH01g0411700 & MORC family $\mathrm{CW}$-type zinc finger protein 3 & 4.92 \\
\hline 13 & MH05g0540900 & Ketol-acid reductoisomerase & $2.44^{*}$ \\
\hline 19 & MH11g0317300 & putative reverse transcriptase & 3.12 \\
\hline 33 & MH04g0636300 & Aminomethyltransferase & $2.21^{*}$ \\
\hline
\end{tabular}


$\begin{array}{lll}38 & \text { MH01g0759700 } \quad \text { Ubiquitin-like specific protease } 1 & 2.02\end{array}$

57 MH07g0520900 Chromodomain-helicase-DNA-binding protein $1 \quad 4.83$

3 a Spot numbers correspond to the protein spot numbers in Fig. S2. "+" Proteins that showed increased abundance after 24 h of

4 heat stress. * The 18 proteins that showed the same abundance patterns (increased or decreased) as the corresponding gene

5 expression levels (up- or down-regulated). 